\title{
An Analysis of the Population of Extended Main Sequence Turn-off Clusters in the Large Magellanic Cloud
}

\author{
Andrés E. Piatti ${ }^{1,2 \star}$ and Nate Bastian ${ }^{3}$ \\ ${ }^{1}$ Observatorio Astronómico, Universidad Nacional de Córdoba, Laprida 854, 5000, Córdoba, Argentina \\ ${ }^{2}$ Consejo Nacional de Investigaciones Científicas y Técnicas, Av. Rivadavia 1917, C1033AAJ, Buenos Aires, Argentina \\ ${ }^{3}$ Astrophysics Research Institute, Liverpool John Moores University, 146 Brownlow Hill, Liverpool L3 5RF, UK
}

Accepted XXX. Received YYY; in original form ZZZ

\begin{abstract}
We combine a number of recent studies of the extended main sequence turn-off (eMSTO) phenomenon in intermediate age stellar (1 - 2 Gyr) clusters in the Large Magellanic Cloud (LMC) in order to investigate its origin. By employing the largest sample of eMSTO LMC clusters so far used, we show that cluster core radii, masses, and dynamical state are not related to the genesis of eMSTOs. Indeed, clusters in our sample have core radii, masses and age-relaxation time ratios in the range $\approx 2-6 \mathrm{pc}, 3.35-$ $5.50\left(\log \left(M_{c l s} / M_{\odot}\right)\right.$ and $0.2-8.0$, respectively. These results imply that the eMSTO phenomenon is not caused by actual age spreads within the clusters. Furthermore, we confirm from a larger cluster sample recent results including young eMSTO LMC clusters, that the FWHM at the MSTOs correlates most strongly with cluster age, suggesting that a stellar evolutionary effect is the underlying cause.
\end{abstract}

Key words: techniques: photometric - galaxies: individual: LMC - Magellanic Clouds.

\section{INTRODUCTION}

It is now clear that a large fraction of studied massive stellar clusters in the Large Magellanic Cloud (LMC) host stellar populations which display a spread in the main sequence turn-off (MSTO) that cannot be explained by photometric errors or stellar binarity (e.g. Milone et al. 2009). From these extended MSTOs (eMSTOs), one can infer age spreads within the clusters, of the order of 100-700 Myr (e.g. Mackey et al. 2008b; Rubele et al. 2011, 2013). If such age spreads are real, this would dramatically affect our understanding of the formation of stellar clusters, which are generally thought to be classic simple stellar populations (SSPs). In addition to actual age spreads, stellar evolutionary effects (e.g., due to stellar rotation) have been invoked to explain the eMSTOs, which may mimic the effect of an age spread (e.g. Bastian \& de Mink 2009; Bastian et al. 2016). Hence, the cause of the eMSTO has received particular attention and support by different research groups.

A number of works have suggested correlations between the inferred age spreads and physical properties of the clusters. The idea behind these suggestions is that some property of the cluster (e.g., mass, radius, density or escape velocity) either determines whether it can undergo extended star formation, or is otherwise related to such an event. For exam-

* E-mail: andres@oac.unc.edu.ar (AEP) ple, Keller et al. (2011) and Keller et al. (2012) have suggested that a large core radius is a by-product of the process by which an eMSTO is formed. In addition, Goudfrooij et al. (2011a,b) and Goudfrooij et al. (2014) have suggested that cluster mass is the property responsible, with only massive clusters (i.e., those with high escape velocities) being able to form multiple generations of stars.

Alternatively, other studies have suggested that true age spreads do not exist within the clusters, with the eMSTOs being caused by stellar evolutionary effects (e.g. Bastian et al. 2016). In this case, we would not expect correlations with a physical property of the clusters, but instead would expect a correlation between cluster age and the inferred age spread (Brandt \& Huang 2015). In fact, such a correlation exists which is consistent with the predictions of rotating stellar isochrones (Niederhofer et al. 2015b,a). The observed correlation between the inferred age spread and the age of the cluster is the strongest evidence so far against the true age spread scenario. Additionally, a number of studies have searched for multiple star-forming events in younger massive clusters, in which such age spreads should be extremely clear, and to date, no evidence for such extended star-formation histories have been found (e.g. Bastian et al. 2013; Cabrera-Ziri et al. 2014),(Cabrera-Ziri et al. 2016; Niederhofer et al. 2016).

In this paper we combine the catalogues of Goudfrooij et al. (2014) of massive clusters in the LMC hosting eM- 
STOs with that from our own studies (Piatti 2013; Piatti et al. 2014; Piatti \& Bastian 2016), in order to bring more evidence about that the eMSTO is linked to stellar evolutionary origins. In $\S 2$ we introduce the data sets and used them to derive cluster structural parameters and masses to explore whether cluster properties are responsible for the eMSTO phenomenon. We discuss the results of our analysis in $\S 3$. Our conclusions are presented in $\S 4$.

\section{CLUSTER ASTROPHYSICAL PROPERTIES}

\subsection{Structural parameters}

The cluster sample analysed here consists in 14 LMC clusters observed by Piatti et al. (2014), 2 LMC clusters studied by Piatti \& Bastian (2016) and SL 529 (Piatti 2013). Sixteen clusters were imaged with the Gemini telescope through the GMOS-S instrument and the $g, i$ filters attached. SL 529 was observed through the $g, r$ filters instead. These set of observations represent an homogeneous sample not only from an observational point of view, but also because the cluster ages and related fundamental parameters were derived using the same techniques and procedures.

Piatti et al. (2014) presented their photometric data sets with the aim of investigating the frequency of occurrence of the eMSTO phenomenon in the LMC cluster population through the link of eMSTOs to large core radii (Keller et al. 2012). They selected an age- and luminosity-limited sample with the original aim of examining if the formation of multiple populations is a general phase of cluster evolution, one possibly related to the puzzling multiple populations seen in ancient Galactic globular clusters (see, for example Carretta et al. 2010). The detailed analysis on the presence or absence of eMSTOs and their connexion to large core radii in these clusters is conducted in this work. On the other hand, the 2 LMC clusters studied by Piatti \& Bastian (2016) and SL 529 have confirmed eMSTOs, and were simply added to the analysed cluster sample.

We derive cluster structural parameters for those clusters in Piatti et al. (2014) and SL 529 from a fully consistent method to that described by Piatti \& Bastian (2016) for the low-mass LMC clusters LW 477 and LW 483. Briefly, we determined the geometrical centres of the clusters by fitting Gaussian distributions to the star counts in the $x$ and $y$ directions, as well as directly on their deepest $g, i$ images, and recovered independent cluster central coordinates closer than \pm 10 pixels $(\sim 1.5$ arcsec $)$. We used the resulting centres to checked whether the clusters exhibit some elliptical signature and derived ellipticity values in the range $0.00-0.20$ \pm 0.05 with an average of $0.08 \pm 0.06$.

For the clusters in our sample, we built the cluster stellar density profiles -expressed as number of stars per $\operatorname{arcsec}^{2}$ - from the completeness corrected star counts performed using the cluster photometric catalogues by Piatti (2013) and Piatti et al. (2014). The background corrected stellar density profiles were fitted using both King (1962) and Plummer (1911) models; the later is used to derived an independent estimate of each clusters half-mass radius. The resulting density profiles are shown in Fig.1. In the figure, we represented the constructed and background subtracted stellar density profiles with open and filled circles, respectively.
Errorbars represent Poisson errors, to which we added the rms error of the background star count to the background subtracted density profiles. The derived core $\left(r_{c}\right)$, half-mass $\left(r_{h}\right)$ and tidal $\left(r_{t}\right)$ radii are listed in Table 1 . As is seen in Fig. 2, there is an overall very good agreement of our core, half-mass and tidal radii with those derived by Correnti et al. (2014) and Goudfrooij et al. (2014) (open circles) for a total of 6 clusters in common (NGC 2173, 2203, 2209, 2213, 2249 and Hodge 6). NGC 2203 is the most discrepant point in the $r_{c}$ panel, although its $r_{h}$ value agrees well with previous determinations. The $r_{t}$ panel shows relatively more scatter, which could be due to the fact that the HST field is not wide enough as to trace accurately the outer cluster regions. We also compared our $r_{c}$ values with those of Mackey \& Gilmore (2003) (open boxes) for 7 clusters in common, namely: NGC 2155, 2162, 2173 (red), 2209 (magenta), 2213 (blue), 2231 and 2249 (orange).

The masses of the clusters in our sample were derived by comparing the observed integrated magnitude of each cluster (corrected for distance modulus and extinction) to that of the Marigo et al. (2008) SSP models with a Kroupa (2002) stellar initial mass function and a metallicity of $Z=$ 0.008 of the appropriate age. The errors in the mass estimate are driven largely by the choice of models (which were also used in estimating the ages). We estimate the uncertainty in the mass to be $\sigma\left(\log \left(M_{c l s} / M_{\odot}\right)\right) \sim 0.3$ dex, i.e., about a factor of two. From the derived masses we estimated both the Jacobi tidal radius $\left(r_{J}\right.$, Chernoff \& Weinberg 1990) and the half-mass relaxation time $\left(t_{r}\right.$, Spitzer \& Hart 1971) of each cluster. We refer the reader to Piatti \& Bastian (2016) for details on the values of the various parameters involved. Table 1 lists the resulting $M_{c l s}, r_{J}$ and $t_{r}$ values. The latter are in excellent agreement with those coming from using Fig. 20 of Pijloo et al. (2015). Indeed, we found no difference between them, independently if Pijloo et al. (2015)'s ones were interpolated by considering scenarios with and without mass segregation. Additionally, we found a half-mass density range of 3-95 $M_{\odot} \mathrm{pc}^{3}$ for the cluster sample. These value are much larger than the minimum density a cluster needs to have in order to be stable against the tidal disruption of a galaxy $\left(\sim 0.1 M_{\odot} \mathrm{pc}^{3}\right.$, Bok (1934)). Accordingly, Wilkinson et al. (2003) also showed that the tidal field of the LMC does not cause any perturbation on the clusters. For the sake of the readers Fig. 3 shows the spatial distribution of the cluster sample. By comparing the resulting $r_{t}$ and $r_{J}$ values, we found however that SL 244, 505, 674, 769 and LW 477 have tidal radii larger than the respective Jacobi's ones (see Glatt et al. 2011).

\subsection{Cluster eMSTOs}

The width of the cluster MSTOs were measured following the precepts outlined in Goudfrooij et al. (e.g. 2011a), i.e., by building a histogram of the number of cluster stars previously decontaminated from field stars- located within a strip running perpendicular to the cluster MSs at the TO positions (see also Piatti 2013; Piatti \& Bastian 2016; Bastian et al. 2016). Such a direction is called the MSTO axis. The cluster MSTOs were cleaned from field stars by using the field star cleaning procedure introduced by $\mathrm{Pi}$ atti \& Bica (2012) and successfully applied in different star field crowdness conditions (e.g. Piatti 2014; Piatti et al. 

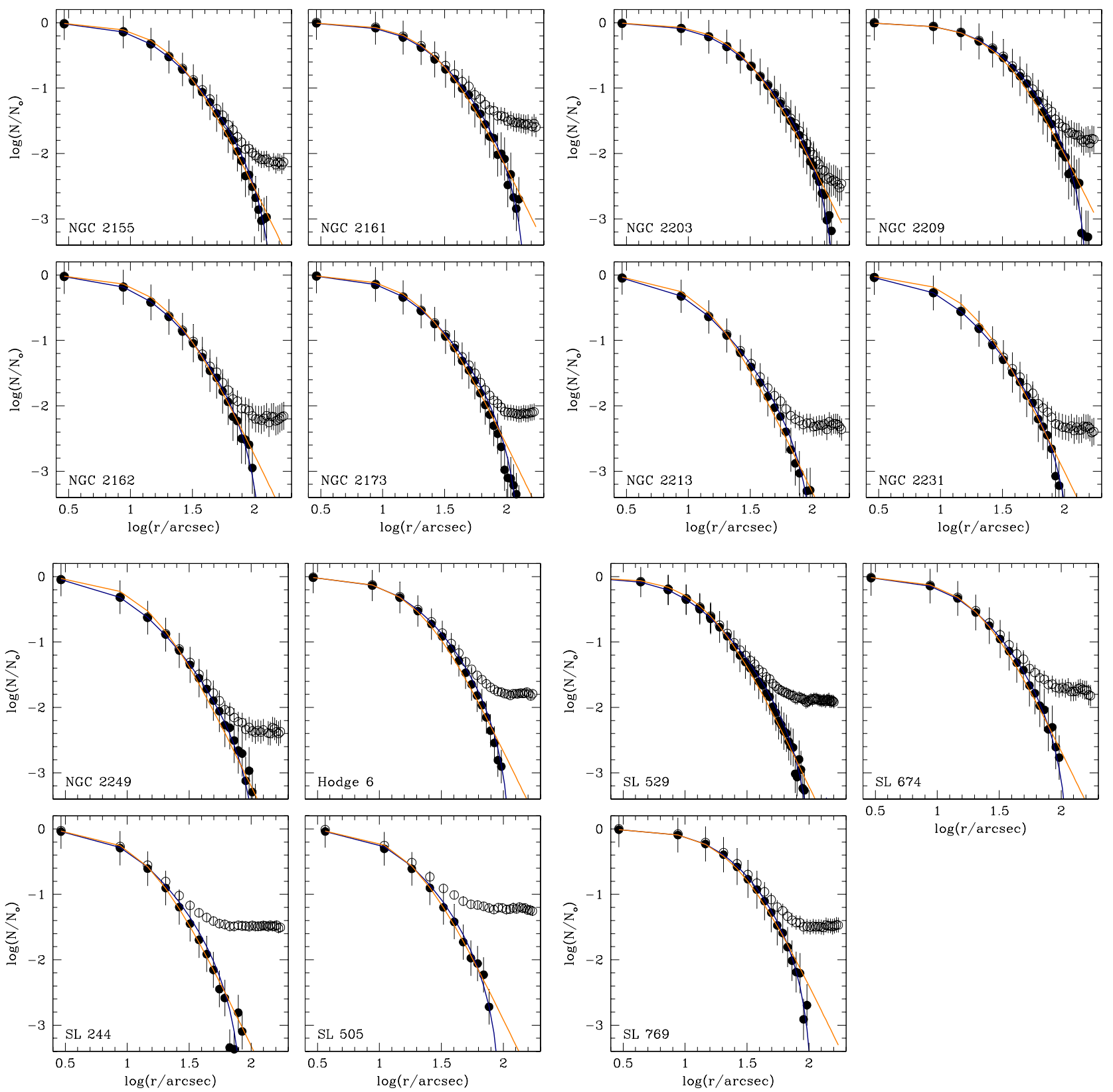

Figure 1. Stellar density cluster profiles obtained from star counts for the cluster sample. Open and filled circles refer to measured and background subtracted surface brightness profiles, respectively. Blue and orange solid lines depict the fitted King and Plummer curves, respectively.

2015; Piatti 2016, and references therein). The left panel of Fig. 4 illustrates these procedures, in which we show the field star cleaned CMD of NGC 2203 and a field star CMD with the same area represented by black and green dots, respectively. The magenta parallelogram represents the MSTO strip. When building the histograms we have taken into account the projection of the photometric errors of each star along the MSTO axis by using a density estimator algorithm descrided in, for instance, Piatti \& Geisler (2013); thus reproducing the real spread caused by photometric uncertainties. We also built MSTO histograms using the same density estimator algorithm without considering photometric errors. When the latter are compared to those including the effects of photometric uncertainties we found similar distributions. Fig. 5 depicts both histograms for the cluster sample, which suggests that the observed MSTO width is not driven by photometric errors. We recall that the data sets of the studied clusters were obtained from identical observational setups (same telescope, detector, filters) and show tight similitudes of completeness and photometric error distributions as LW 477 and LW 483 (Piatti \& Bastian 2016, see their Fig. $5)$.

We then fitted Gaussian functions to the cluster MSTO 
Table 1. Fundamental properties of LMC star clusters.

\begin{tabular}{|c|c|c|c|c|c|c|c|c|c|c|}
\hline Star cluster & $\log (t)$ & $\begin{array}{c}r_{c} \\
(\mathrm{pc})\end{array}$ & $\begin{array}{c}r_{h} \\
(\mathrm{pc})\end{array}$ & $\begin{array}{c}r_{t} \\
(\mathrm{pc})\end{array}$ & $\begin{array}{c}r_{J} \\
(\mathrm{pc})\end{array}$ & $\begin{array}{c}M_{c l s} \\
\left(10^{3} M_{\odot}\right)\end{array}$ & $\begin{array}{c}t_{r} \\
(\mathrm{Myr})\end{array}$ & $\begin{array}{c}F W H M_{\mathrm{MSTO}} \\
(\mathrm{Myr})\end{array}$ & $\begin{array}{l}\text { eMSTO }_{\text {lit }} \\
(\mathrm{Myr})\end{array}$ & Ref. \\
\hline NGC 2155 & $9.50 \pm 0.05$ & $3.6 \pm 0.2$ & $7.6 \pm 0.3$ & $36.4 \pm 2.4$ & 48.6 & $53.0 \pm 36.6$ & 985 & $130 \pm 127$ & & \\
\hline NGC 2161 & $9.35 \pm 0.05$ & $4.8 \pm 0.2$ & $9.1 \pm 0.3$ & $36.4 \pm 2.4$ & 42.2 & $22.0 \pm 15.2$ & 925 & $208 \pm 45$ & & \\
\hline NGC 2162 & $9.20 \pm 0.05$ & $3.2 \pm 0.2$ & $6.6 \pm 0.3$ & $29.1 \pm 2.4$ & 55.1 & $37.5 \pm 25.9$ & 702 & $225 \pm 25$ & & \\
\hline NGC 2173 & $9.25 \pm 0.05$ & $3.6 \pm 0.2$ & $7.2 \pm 0.3$ & $32.7 \pm 2.4$ & 51.3 & $63.9 \pm 44.2$ & 996 & $342 \pm 49$ & 431 & 2 \\
\hline NGC 2203 & $9.30 \pm 0.05$ & $4.8 \pm 0.2$ & $9.4 \pm 0.3$ & $38.8 \pm 2.4$ & 87.9 & $111.1 \pm 76.7$ & 1855 & $349 \pm 15$ & 475 & 2 \\
\hline NGC 2209 & $9.15 \pm 0.05$ & $6.1 \pm 0.2$ & $10.4 \pm 0.3$ & $38.8 \pm 2.4$ & 47.5 & $26.2 \pm 18.1$ & 1202 & $371 \pm 41$ & 230 & 1 \\
\hline NGC 2213 & $9.25 \pm 0.05$ & $2.2 \pm 0.2$ & $4.7 \pm 0.3$ & $26.7 \pm 2.4$ & 46.6 & $42.4 \pm 29.3$ & 445 & $356 \pm 26$ & 329 & 2 \\
\hline NGC 2231 & $9.20 \pm 0.05$ & $2.4 \pm 0.2$ & $5.7 \pm 0.3$ & $29.1 \pm 2.4$ & 45.9 & $25.7 \pm 17.8$ & 481 & $300 \pm 17$ & & \\
\hline NGC 2249 & $9.15 \pm 0.05$ & $2.2 \pm 0.2$ & $5.0 \pm 0.3$ & $29.1 \pm 2.4$ & 44.6 & $21.0 \pm 14.5$ & 372 & $174 \pm 20$ & 450 & 1 \\
\hline Hodge 6 & $9.40 \pm 0.10$ & $3.9 \pm 0.2$ & $6.9 \pm 0.3$ & $29.1 \pm 2.4$ & 33.4 & $79.9 \pm 55.2$ & 1019 & $235 \pm 75$ & 238 & 2 \\
\hline SL 244 & $9.40 \pm 0.10$ & $2.4 \pm 0.2$ & $4.7 \pm 0.3$ & $21.8 \pm 2.4$ & 11.2 & $24.5 \pm 16.9$ & 358 & $285 \pm 27$ & & \\
\hline SL 505 & $9.30 \pm 0.10$ & $3.2 \pm 0.2$ & $6.0 \pm 0.3$ & $24.2 \pm 2.4$ & 13.1 & $10.9 \pm 7.5$ & 374 & $458 \pm 41$ & & \\
\hline SL 529 & $9.35 \pm 0.05$ & $2.4 \pm 0.2$ & $5.0 \pm 0.3$ & $26.7 \pm 2.4$ & 31.1 & $10.0 \pm 6.9$ & 280 & $406 \pm 82$ & & \\
\hline SL 674 & $9.45 \pm 0.05$ & $3.6 \pm 0.2$ & $6.9 \pm 0.3$ & $29.1 \pm 2.4$ & 22.7 & $15.7 \pm 10.9$ & 536 & $204 \pm 70$ & & \\
\hline SL 769 & $9.35 \pm 0.10$ & $4.8 \pm 0.2$ & $8.2 \pm 0.3$ & $26.7 \pm 2.4$ & 21.5 & $17.7 \pm 12.2$ & 721 & $336 \pm 83$ & & \\
\hline LW 477 & $9.10 \pm 0.05$ & $2.7 \pm 0.5$ & $6.3 \pm 0.6$ & $26.7 \pm 2.4$ & 21.6 & $2.2 \pm 1.5$ & 155 & $308 \pm 25$ & & \\
\hline LW 483 & $9.10 \pm 0.05$ & $2.7 \pm 0.5$ & $6.3 \pm 0.6$ & $29.1 \pm 2.4$ & 32.0 & $5.5 \pm 3.8$ & 163 & $299 \pm 21$ & & \\
\hline \multicolumn{11}{|c|}{ Additional clusters taken from the literature } \\
\hline NGC 411 & $9.16 \pm 0.02$ & $4.23 \pm 0.26$ & $6.12 \pm 0.79$ & & & $4.67 \pm 0.03$ & & & 516 & 2 \\
\hline NGC 419 & $9.16 \pm 0.02$ & $5.48 \pm 2.01$ & $7.67 \pm 2.86$ & & & $5.38 \pm 0.08$ & & & 560 & 2 \\
\hline NGC 1651 & $9.30 \pm 0.02$ & $4.57 \pm 0.36$ & $12.82 \pm 2.01$ & & & $4.91 \pm 0.06$ & & & 315 & 2 \\
\hline NGC 1718 & $9.25 \pm 0.02$ & $3.74 \pm 0.24$ & $5.42 \pm 0.56$ & & & $4.83 \pm 0.07$ & & & 406 & 2 \\
\hline NGC 1751 & $9.15 \pm 0.02$ & $5.76 \pm 0.41$ & $7.10 \pm 0.87$ & & & $4.81 \pm 0.06$ & & & 353 & 2 \\
\hline NGC 1755 & $7.9 \pm 0.05$ & 1.7 & & & & 4.0 & & & 25 & $6,7,8$ \\
\hline NGC 1783 & $9.23 \pm 0.02$ & $10.50 \pm 0.49$ & $11.40 \pm 2.24$ & & & $5.42 \pm 0.11$ & & & 403 & 2 \\
\hline NGC 1795 & $9.15 \pm 0.05$ & $4.13 \pm 0.61$ & $7.47 \pm 1.23$ & & & $4.50 \pm 0.07$ & & & 350 & 1 \\
\hline NGC 1806 & $9.20 \pm 0.02$ & $5.91 \pm 0.27$ & $9.04 \pm 1.24$ & & & $5.10 \pm 0.06$ & & & 370 & 2 \\
\hline NGC 1846 & $9.23 \pm 0.02$ & $8.02 \pm 0.49$ & $8.82 \pm 0.68$ & & & $5.24 \pm 0.09$ & & & 567 & 2 \\
\hline NGC 1850 & $8.00 \pm 0.05$ & 2.7 & 11.2 & & & 5.3 & & & 44.4 & 4,5 \\
\hline NGC 1852 & $9.15 \pm 0.02$ & $5.10 \pm 0.46$ & $6.97 \pm 0.83$ & & & $4.66 \pm 0.07$ & & & 312 & 2 \\
\hline NGC 1856 & $8.48 \pm 0.10$ & $3.18 \pm 0.12$ & $8.00 \pm 0.90$ & & & $5.00 \pm 1.00$ & & & 150 & 3 \\
\hline NGC 1978 & 9.3 & 4.3 & 9.6 & & & 5.5 & & & $<100$ & $2,9,10,11$ \\
\hline NGC 1987 & $9.04 \pm 0.02$ & $4.18 \pm 0.46$ & $12.78 \pm 3.05$ & & & $4.74 \pm 0.04$ & & & 234 & 2 \\
\hline NGC 2108 & $9.00 \pm 0.025$ & $5.42 \pm 0.27$ & $7.20 \pm 0.76$ & & & $4.71 \pm 0.07$ & & & 230 & 2 \\
\hline NGC 2154 & $9.19 \pm 0.02$ & $4.50 \pm 0.29$ & $5.69 \pm 0.51$ & & & $4.61 \pm 0.06$ & & & 431 & 2 \\
\hline LW 431 & $9.28 \pm 0.02$ & $4.03 \pm 0.24$ & $9.10 \pm 3.16$ & & & $4.56 \pm 0.07$ & & & 277 & 2 \\
\hline Hodge 2 & $9.35 \pm 0.02$ & $2.67 \pm 0.41$ & $9.09 \pm 2.33$ & & & $4.70 \pm 0.07$ & & & 363 & 2 \\
\hline IC 2146 & $9.28 \pm 0.05$ & $8.89 \pm 1.36$ & $12.53 \pm 1.92$ & & & $4.49 \pm 0.07$ & & & 410 & 1 \\
\hline
\end{tabular}

References: 1) Correnti et al. (2014); 2) Goudfrooij et al. (2014); 3) Milone et al. (2015); 4) McLaughlin \& van der Marel (2005); 5) Bastian et al. (2016); 6) Elson et al. (1989); 7) Popescu et al. (2012); 8) Milone et al. (2016); 9) Fischer et al. (1992); 10) Milone et al. (2009); 11) Mucciarelli et al. (2007).

histograms using the IRAF ${ }^{1}$ NGAUSSFIT task and the results are overplotted on Fig. 5 using black lines.

1 IRAF is distributed by the National Optical Astronomy Observatories, which is operated by the Association of Universities for Research in Astronomy, Inc., under contract with the National Science Foundation.

\subsection{Synthetic cluster experiments}

In order to estimate the true spread across the MSTO we need to correct for the effects of photometric errors and stellar binarity. To do so, we created artificial clusters using the same isochrones and distance moduli that were used in the observational analysis of the clusters in our sample. We stochastically selected 10,000 stars from a Salpeter (1955) initial mass function between $1 \mathrm{M}_{\odot}$ and the maximum stellar mass still alive at a given age. We then added photometric errors as well as the effects of binarity, where we assumed a flat binary distribution. In order to estimate the photometric errors and binary fraction we compared the width and distribution of stars across the (verticalised) main sequence (Piatti \& Bastian 2016). The photometric error was estimated by fitting a Gaussian to the main peak of the distribution, while the binary fraction was estimated from the 


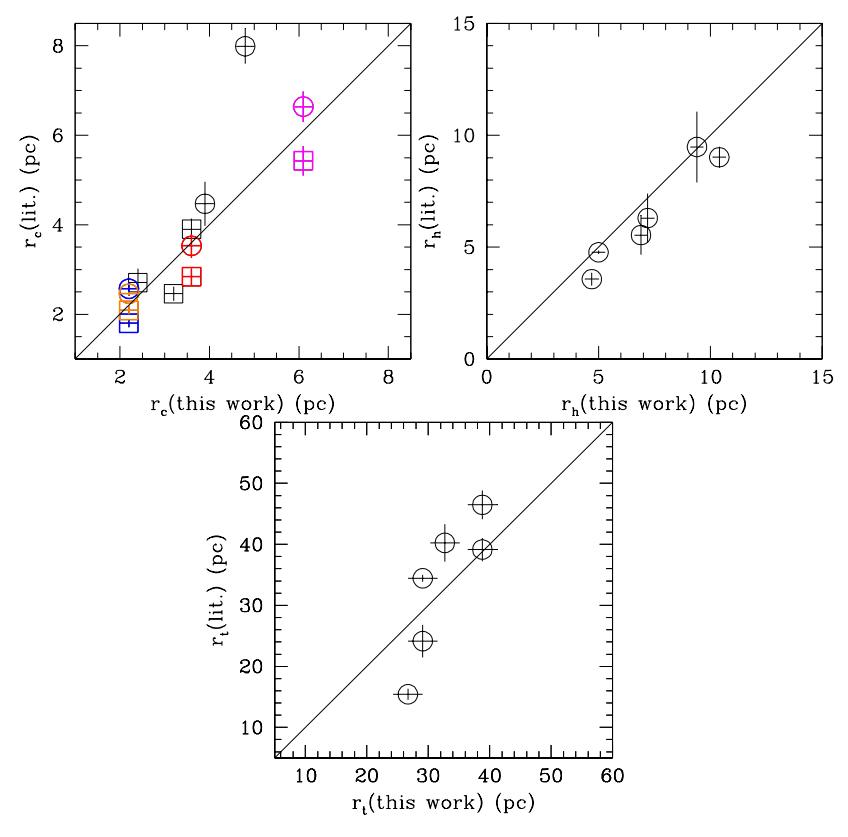

Figure 2. Comparison of structural parameters derived in this work with those published by Correnti et al. (2014) and Goudfrooij et al. (2014) are drawn with open circles. Errorbars for the individual values are also drawn. The solid lines represent the identity relation. Core radii derived by Mackey \& Gilmore (2003) are represented by open boxes. Coloured symbols refer to NGC 2173 (red), 2209 (magenta), 2213 (blue) and 2249 (orange).

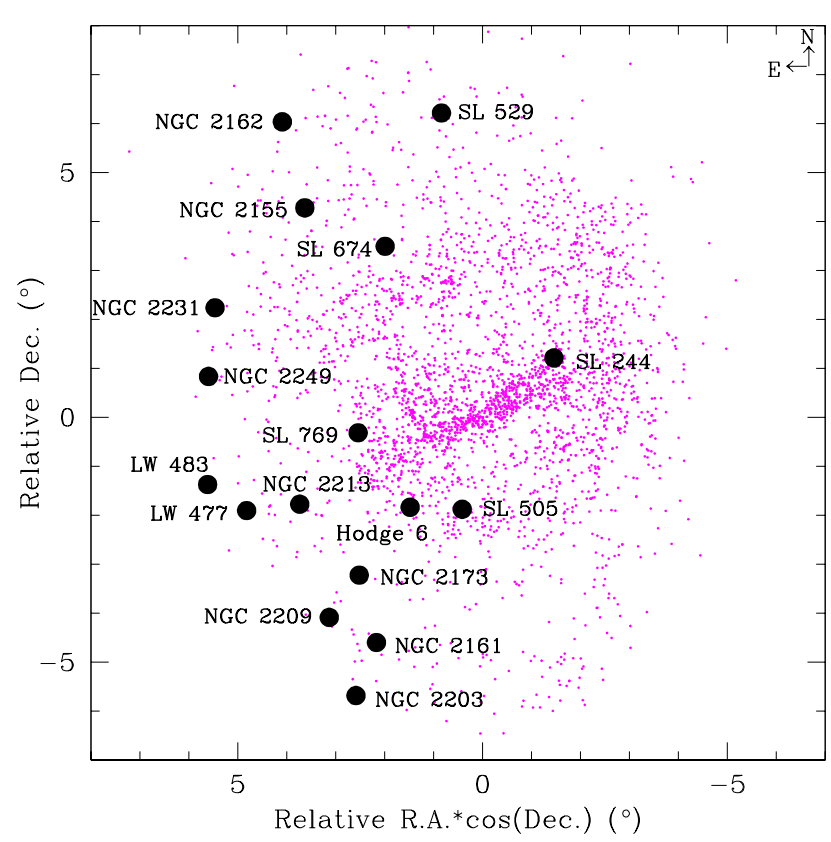

Figure 3. Spatial distribution of the Bica et al. (2008)'s catalogue of star clusters in the LMC centred at R.A. $=05^{h} 23^{m} 34^{s}$, Dec. $=-69^{\circ} 45^{\prime} 22^{\prime \prime}$ (J2000), projected onto the sky, in magentacoloured filled circles. The studied clusters are highlighted with black filled circles. extended tail of the distribution to redder colours. We found that the observed distributions were well fit with simulations that had a photometric error in magnitude of $0.035 \mathrm{mag}$ in both the $g$ and $i$ filters. Our experiments led to high estimates of the binary fraction (25\% binaries with mass ratios above 0.67), which is about twice that found in Milone et al. (2009). We note that by adopting potentially higher binary fractions than are actually present, we will overestimate the inferred age spread of the synthetic cluster (i.e., SSP) which will cause our estimated age spreads of the observations to be underestimated. However, in most cases this will not lead to a large change in the estimate spreads.

Each synthetic cluster was then analysed in the same way as the observed clusters. An example is shown in Fig. 4, where in the middle panel the synthetic cluster stars are shown in red and the resulting inferred age distribution is shown in red in the right panel. For each observed cluster, we estimated the inferred age spread by approximating the observed and modelled (as an SSP of the best fitting age) age distributions as Gaussians, and subtracted the modelled width $\left(\sigma_{\mathrm{SSP}}\right)$ from the observed width $\left(\sigma_{\mathrm{obs}}\right)$ in quadrature to obtain the intrinsic inferred width $\left(\sigma_{\text {intrinsic }}\right)$. Fig. 6 shows the observed (cleaned from field stars), modelled and true $F W H M_{\mathrm{MSTO}}$ as a function of the cluster age, depicted with blue open triangles, red open boxes and black filled circles, respectively.

We note that our estimated widths are in good agreement with that estimated in the literature (based mostly on HST observations), but there are some outliers. In particular, our estimate of the width of NGC 2249 ( 170 Myr) is significantly less than that found by Correnti et al. (2014, $\sim 450 \mathrm{Myr}$ ). Part of this difference may be due to the fact that we assign a larger age $(1.4 \mathrm{Gyr})$ to the cluster than Correnti et al. (1 Gyr). However, we note that such a large inferred age spread is clearly incompatible with our observed CMD of this cluster.

As a test of the adopted method, we have estimated the inferred age spread of NGC 2203, based on the HST/WFC3 photometric catalogue of Goudfrooij et al. (2014). We find an age spread of $580 \mathrm{Myr}$ (without correcting for the effects of photometric errors or binarity), in decent agreement with that found by Goudfrooij et al. (2014) and Niederhofer et al. (2016), although significantly larger than we found based on our ground based data $(\sim 350 \mathrm{Myr})$. The source of this discrepancy is unknown. We speculate in the possibility that the larger GMOS-S field of view could allow us to do a better job in cleaning the cluster CMDs from field star contamination. On average, our estimated FWHM of the inferred age spread is $\sim 50$ Myr less than that found in the literature.

For comparison purposes, Table 1 includes in its last columns the $F W H M$ s (eMSTOs) taken from Correnti et al. (2014) and Goudfrooij et al. (2014), respectively. As can be seen, there is a general good agreement with our values, although some differences are expected to arise due to different cluster regions and the use of ground based and space based images. In the following, we use our derived values on a homogeneous basis. 

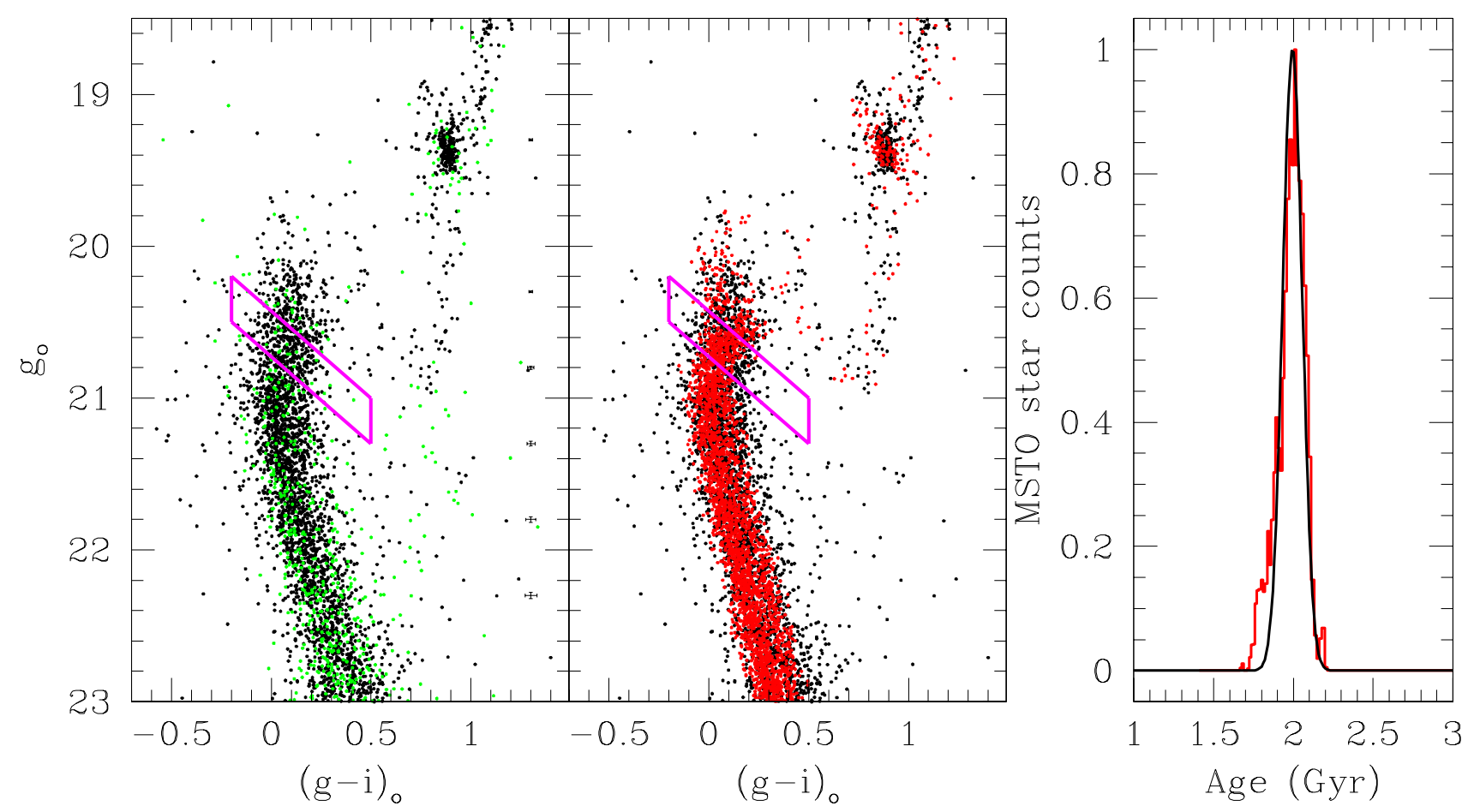

Figure 4. Left panel: Cleaned CMD of NGC 2203 (black dots) and that for the field star CMD (i.e., the background with an equal area) (green dots). The MSTO strip is represented by a magenta parallelogram. Errorbars are also drawn in the right margin. Middle panel: Cleaned and modelled CMDs drawn with black and red dots, respectively. Right panel: The resulting inferred age distribution of synthetic stars located within the MSTO strip (red line) and the respective fitted Gaussian function superimposed (black line).

\section{ANALYSIS AND DISCUSSION}

The cluster parameter values listed in Table 1 allow us to carry out an analysis in the light of some recent results about the origin and astrophysical scenarios in which eMSTOs can arise. We enlarged our sample by adding those clusters analysed by Correnti et al. (2014) and Goudfrooij et al. (2014). We used their ages, $r_{c}, F W H M_{\mathrm{MSTO}}$ and masses. We also included NGC 1755 (Elson et al. 1989; Popescu et al. 2012; Milone et al. 2016), NGC 1850 (McLaughlin \& van der Marel 2005; Bastian et al. 2016) and NGC 1856 (Correnti et al. 2014; Milone et al. 2015), three young LMC clusters with confirmed eMSTOs. We did not include some clusters studied by Milone et al. (2009) because of the lack of known structural parameters. This is the largest cluster sample so far used to study the eMSTO phenomemon. As far as we aware of, it consists of all the available literature data.

Keller et al. (2012, hereafter K12) confirmed results by Keller et al. (2011) in the sense that clusters with eMSTO morphology have the largest core radii at a given age. According to the authors, the correspondence between the large cluster radius and the presence of eMSTOs implies that either the formation of stars subsequent to the primary stars of the cluster is fostered by the environment of a cluster of large core radius or it imparts a kinematic signature that acts to increase the core radius of the host cluster. They drew such conclusions by linking results of Mackey et al. (2008a) - who showed that primordial mass segregation leads to a cluster of $\sim 1-2$ Gyr have a larger core radius than unsegregated ones - and their Fig. 4. Hence, they proposed that the eMSTO phenomenon is a common pathway for massive star clusters.

Fig. 7 shows at the upper-left panel the region where K12 identified eMSTO clusters depicted by a thick contour rectangle. Clusters taken from the literature (Correnti et al. 2014; Goudfrooij et al. 2014) were drawn with solid black boxes to differentiate them from our own cluster sample (large coloured boxes). As can be seen, the marked rectangular area is mostly occupied by clusters previously analysed by K12; NGC $2209\left(\log \left(t \mathrm{yr}^{-1}\right)=9.15\right)$ being the only cluster studied here. NGC 2209 complies with the K12's prediction in the sense that an eMSTO cluster does have a large core radius, since it lies at the top of that rectangular area and observations show that it has an eMSTO (Correnti et al. 2014). Likewise, we here identified other clusters (NGC 2155, 2161, 2173, 2203, Hodge 6, SL 674 and 769) with large core radii exhibiting eMSTOs as shown in the upper-right panel.

However, and perhaps more importantly, we also identify in the upper-right panel a number of eMSTO clusters with smaller core radii (NGC 2162, 2213, 2231, 2249, SL 244, 505, 529, LW 477 and 483). Looking at the upper-left panel, it appears that the eMSTO clusters in our sample spread all along the core radius range at the considered age regime. Notice that K12 considered NGC 2173, 2213 and 2249 as intermediate-age clusters without any hint of eMSTOs, while Goudfrooij et al. (2014, hereafter G14) and Correnti et al. (2014) did confirm their eMSTO nature in good agreement with the present values. Hence, our sample does not confirm that large core radii are related to the eMSTO phenomenon. 


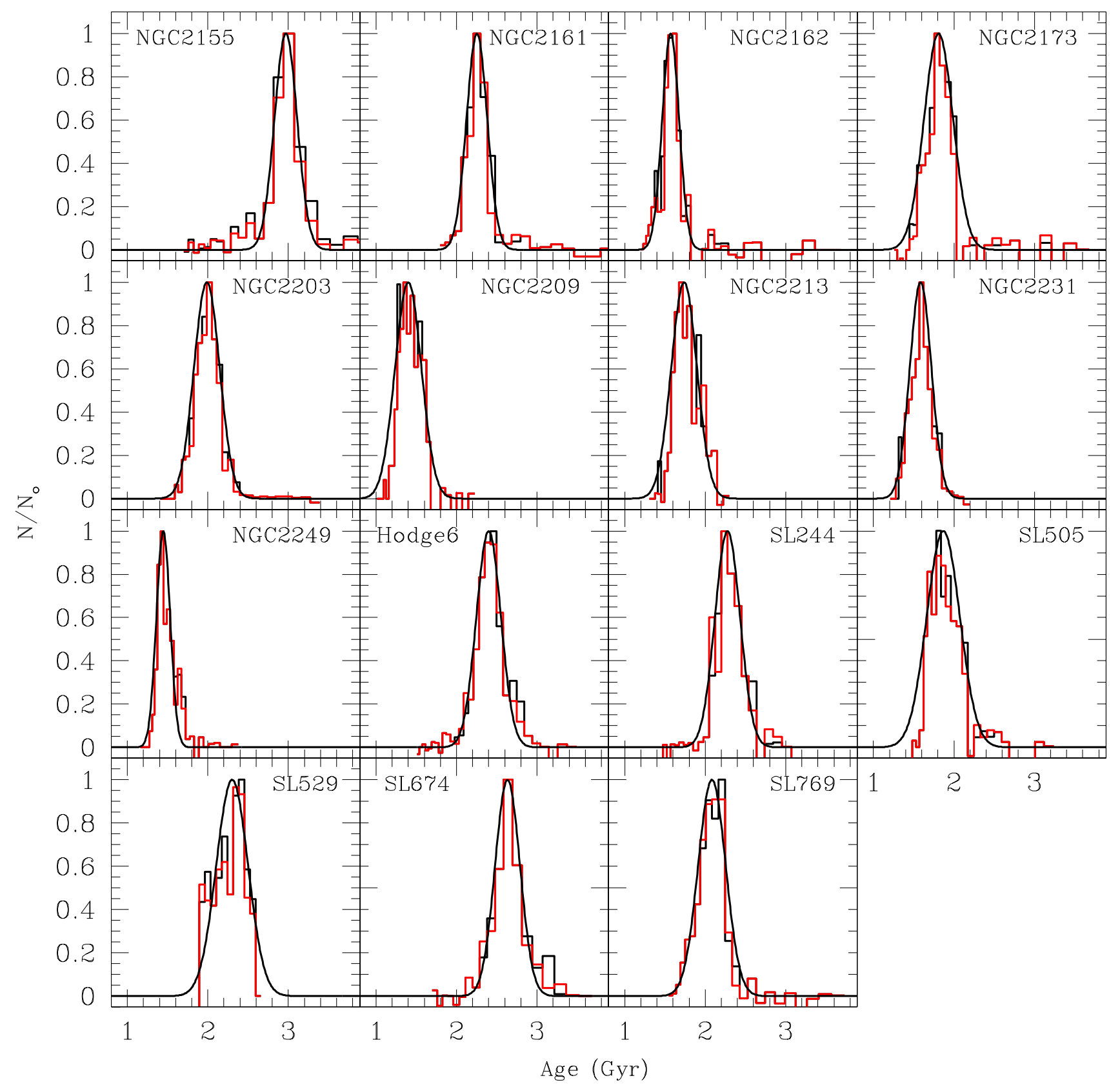

Figure 5. Normalised star counts along the MSTO axis (see Goudfrooij et al. 2011a). Thick black and red histograms refer to the star counts obtained by considering or not the photometric uncertainties, respectively. Gaussian distributions fitted to the black histograms are also superimposed.

K12 also claimed that only clusters of mass similar to, or greater than, those now exhibiting the eMSTO at an age of $1-3$ Gyr $\left(\log \left(M_{\text {cls }} / M_{\odot}\right) \gtrsim 4.8\right)$ possessed a sufficiently deep potential to capture and hold enriched gas for the formation of second-generation stars, and therefore to present eMSTOs. However, the bottom-panels of Fig. 7 suggests not only massive clusters can host eMSTOs.

G14 found that the width of the MSTO region correlates with the cluster's current mass with a probability of the absence of a correlation smaller than 1.3 per cent. They noted, however, that due to different ages and radii clusters underwent different amount of mass loss. Particularly, they applied "correction factors" to boost the initial escape velocity by adopting extreme mass loss and radii expansion. From Table 1, however, we learn rather that only clusters with masses larger than $\log \left(M_{c l s} / M \odot\right) \sim 4.3$ follows the relation fitted by G14 (see their Fig. 5), and that less massive clusters - most of them having relatively large $F W H M_{\mathrm{MSTO}}$ values - are far from that straight line (see also bottom-right panel of Fig. 7). Since cluster masses and escape velocities 


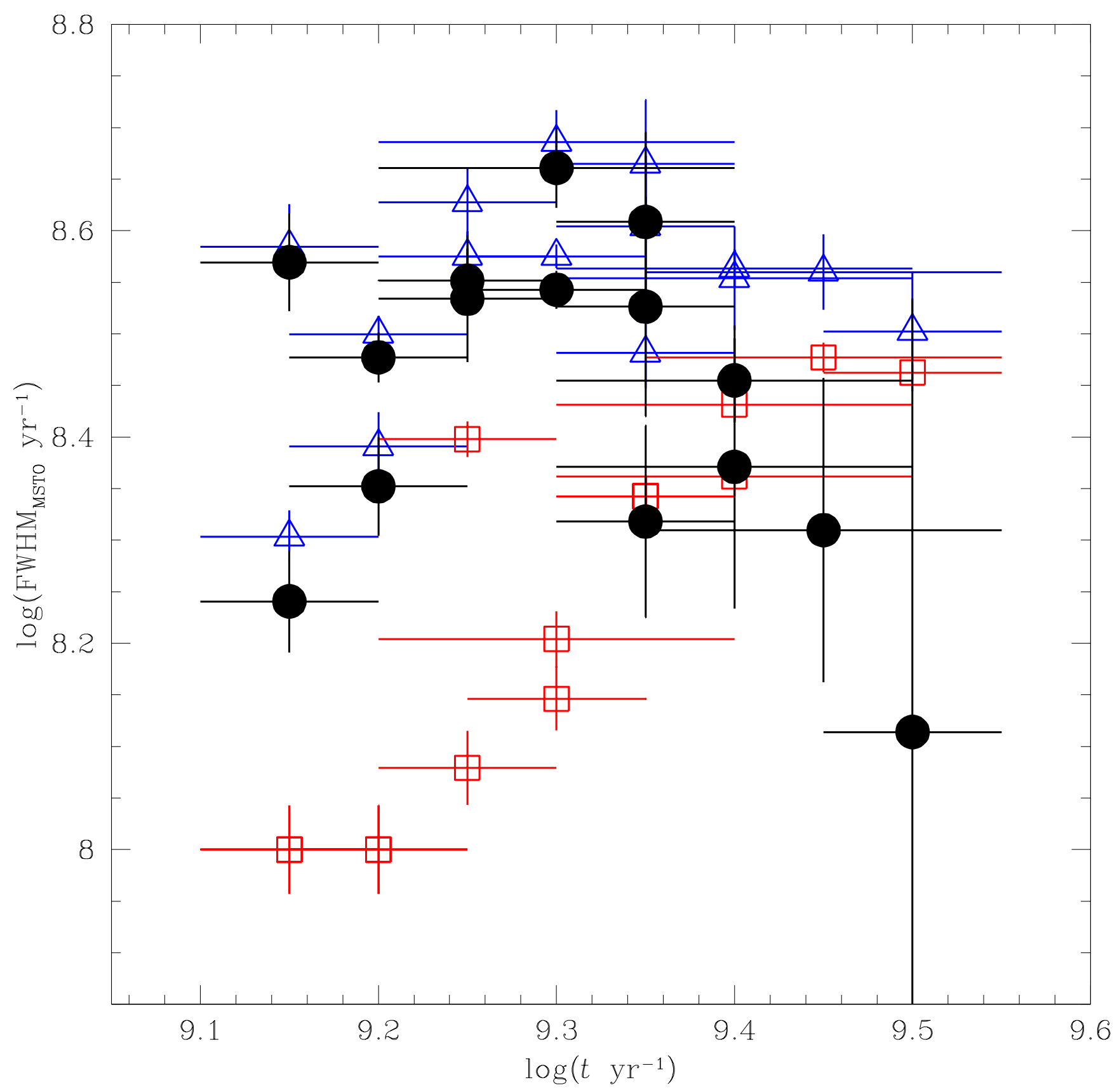

Figure 6. The observed (cleaned from field stars), modelled and true $F W H M_{\mathrm{MSTO}}$ as a function of the cluster age, depicted with blue open triangles, red open boxes and black filled circles, respectively. Errorbars are also drawn.

are largely interchangeable, our results thus contradict argumentations by G14, in the sense that eMSTOs only occur for clusters whose early escape velocities are higher than the wind velocities of stars that provide material from which second-generation stars can form.

In Fig. 8, we show the $F W H M_{\text {MSTO }}$ versus $\log \left(t \mathrm{yr}^{-1}\right)$ for the whole cluster sample. We included the cluster masses as a third parameter, distinguishing them with a colourcoded scale as shown at the right-hand border of the figure. The relationship $F W H M_{\text {MSTO }}$ versus $\log \left(t \mathrm{yr}^{-1}\right)$ seems to follow a inverted- $\mathrm{V}$ trend, peaked at $\log \left(t \mathrm{yr}^{-1}\right) \sim 9.2-9.3$ (age $\sim 2$ Gyr), in very good agreement with Bastian et al. (2016, see their Fig. 4). For clusters younger than $<1$ Gyr there is a stronger correlation as found by Niederhofer et al. (2015a) and discussed at length in Niederhofer et al. (2016). Notice that our Fig. 8 (right-hand panel) reveals that eMSTO features are found in clusters with different relaxation times.

Finally, of particular note is the high mass cluster NGC 1978, which does not show any evidence of an age spread (< 100 Myr Milone et al. 2009). ItâĂŹs mass $\left(\operatorname{logm} / M_{\odot} \sim 5.5\right.$ Goudfrooij et al. 2014) and escape velocity 

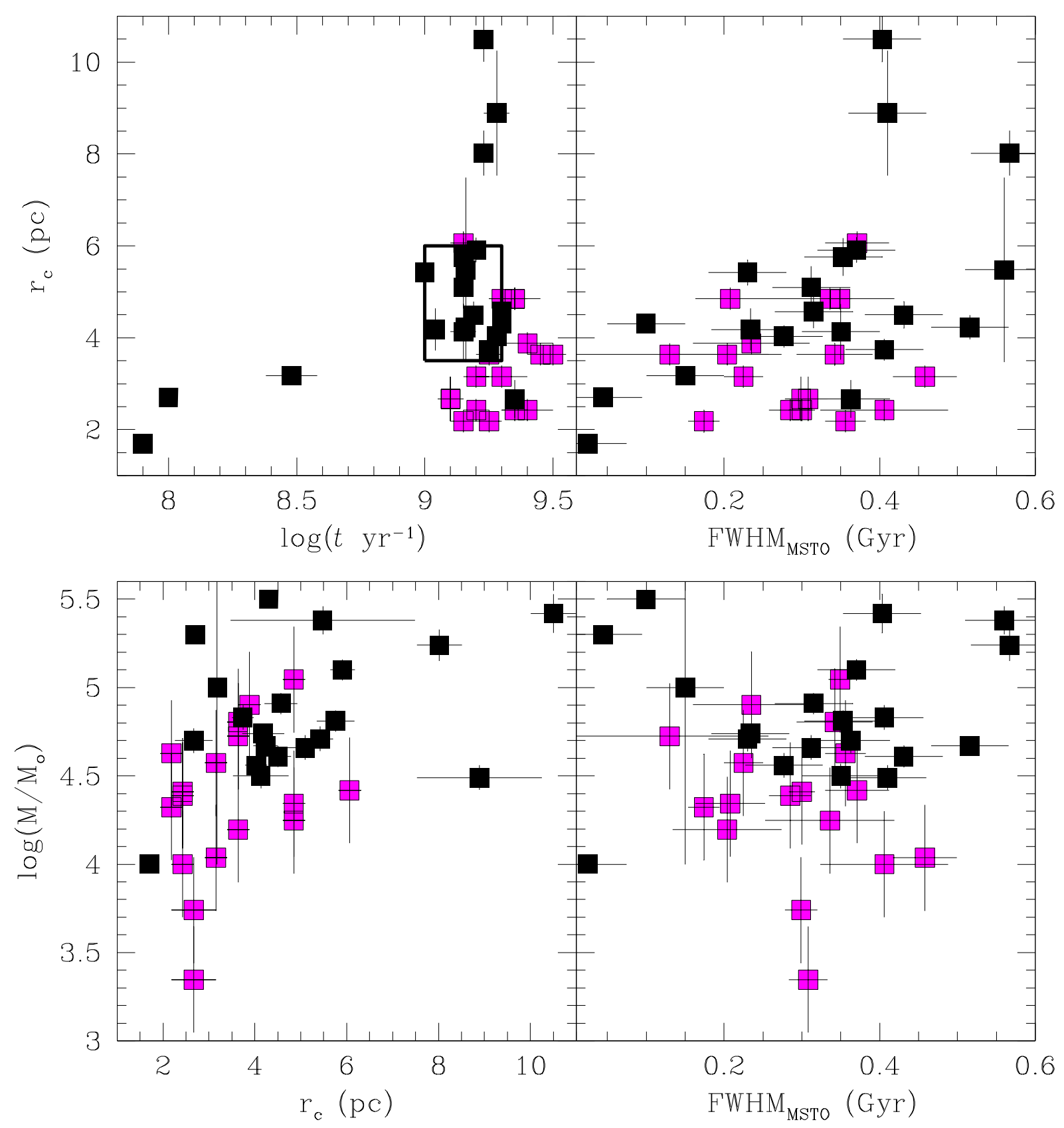

Figure 7. Relationships between various cluster parameters. Black solid boxes represent additional clusters taken from the literature (see Table 1).

suggest that it should show a very large age spread in the G14 scenario, in contradiction to the observed tight eMSTO. Alternatively, since its age is past the "turn-over' in the agedelta(age) plot, it would be expected to have a very narrow MSTO in the stellar rotation scenario, consistent with what is observed. Taken together with the previous evidence, this suggests that actual age spreads are not present in massive clusters.

\section{CONCLUSIONS}

From the photometric data sets of 15 LMC clusters observed by Piatti et al. (2014) with the Gemini-South telescope, we derived their structural parameters, masses, relaxation times and $F W H M_{\mathrm{MSTO}}$ values from a fully consistent method to that described by Piatti \& Bastian (2016) for other two lowmass LMC clusters, also included in our subsequent analysis. The $F W H M_{\mathrm{MSTO}}$ values do not take into acount the effect of stellar binarity and cover the range $\sim 200-500 \mathrm{Myr}$, i.e., they do show eMSTOs. Six clusters in common with 


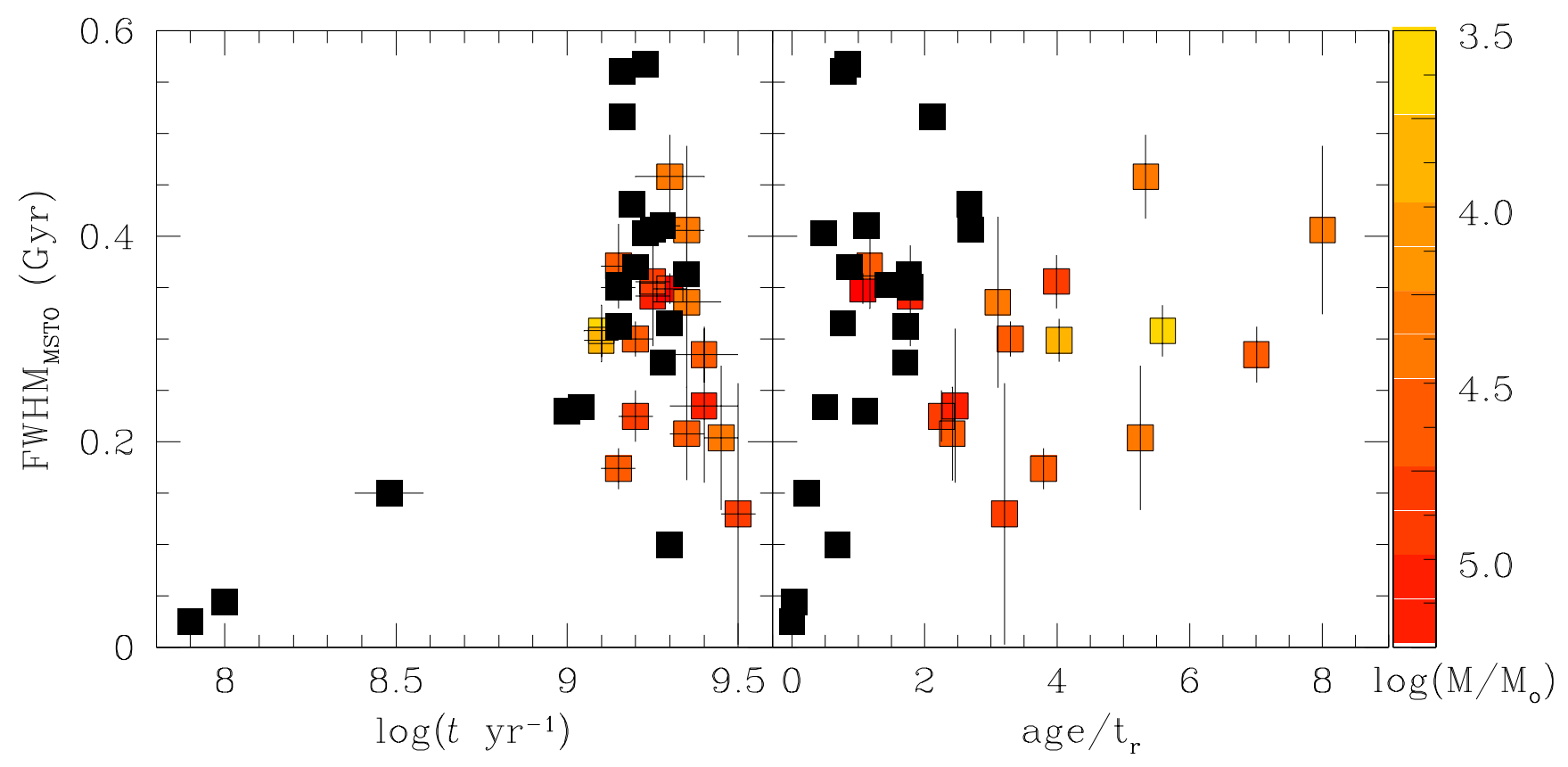

Figure 8. Relationships between various cluster parameters. Black solid boxes represent additional clusters taken from the literature (see Table 1).

Correnti et al. (2014)'s and G14's samples of eMSTO clusters show an overall good agreement of their core radii and

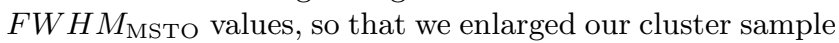
by adding those clusters studied by them. We gathered in total 37 eMSTO LMC clusters. This is the largest cluster sample so far used to study the eMSTO phenomenon.

The derived cluster core radii spread over the range of values $(\approx 4-6 \mathrm{pc}$ ) claimed by K12 to exhibit eMSTOs. However, our whole core radii range reaches values as small as $\sim 2 \mathrm{pc}$, thus providing the first evidence that they are not related to eMSTOs. Since large core radii and large masses are roughly correlated, K12 also suggested a lower mass limit of $\log \left(M_{\text {cls }} / M_{\odot}\right) \sim 4.8$ for eMSTO clusters. Here we found that clusters with masses $\gtrsim 10^{3.35}$ also host eMSTOs. Likewise, this result does not support the scenario suggested by G14 about that eMSTOs only occur for clusters with high early escape velocities, since cluster masses and escape velocities are interchangeable. Moreother, if eMSTOs were orginated by two generations of stars, they should mainly retain second-generation stars after evolving several relaxation times. However, we show that eMSTOs are observed in dynamically relaxed clusters.

Finally, we confirm from a larger cluster sample the type of " $\Lambda$ " distribution in $F W H M_{\text {MSTO versus } \log \left(t \mathrm{yr}^{-1}\right) \text { plane }}$ found by Niederhofer et al. (2016). The way to test if age is the defining parameter of eMSTOs is to look for them at younger clusters (<500 Myr). Indeed, NGC 1755 (Milone et al. 2016, $80 \mathrm{Myr}$ ), NGC 1850 (Bastian et al. 2016, $100 \mathrm{Myr}$ ), NGC 1856 (Correnti et al. 2014, $300 \mathrm{Myr}$ ) show small eMSTOs, consistent with the trend.

\section{ACKNOWLEDGEMENTS}

We thank the anonymous referee whose comments and suggestions allowed us to improve the manuscript. We thank Paul Goudfrooij for providing his photometric catalogues for NGC 2203. NB gratefully acknowledges financial support from the Royal Society (University Research Fellowship) and the European Research Council (ERC-CoG-646928, MultiPop).

\section{REFERENCES}

Bastian N., de Mink S. E., 2009, MNRAS, 398, L11

Bastian N., Cabrera-Ziri I., Davies B., Larsen S. S., 2013, MNRAS, 436, 2852

Bastian N., et al., 2016, MNRAS,

Bok B. J., 1934, Harvard College Observatory Circular, 384, 1

Brandt T. D., Huang C. X., 2015, ApJ, 807, 58

Cabrera-Ziri I., Bastian N., Davies B., Magris G., Bruzual G., Schweizer F., 2014, MNRAS, 441, 2754

Cabrera-Ziri I., et al., 2016, MNRAS, 457, 809

Carretta E., Bragaglia A., Gratton R. G., Recio-Blanco A., Lucatello S., D'Orazi V., Cassisi S., 2010, A\&A, 516, A55

Chernoff D. F., Weinberg M. D., 1990, ApJ, 351, 121

Correnti M., Goudfrooij P., Kalirai J. S., Girardi L., Puzia T. H., Kerber L., 2014, ApJ, 793, 121

Elson R. A. W., Freeman K. C., Lauer T. R., 1989, ApJ, 347, L69

Fischer P., Welch D. L., Mateo M., 1992, AJ, 104, 1086

Glatt K., et al., 2011, AJ, 142, 36

Goudfrooij P., Puzia T. H., Kozhurina-Platais V., Chandar R., 2011a, ApJ, 737, 3

Goudfrooij P., Puzia T. H., Chandar R., Kozhurina-Platais V., 2011b, ApJ, 737, 4

Goudfrooij P., et al., 2014, ApJ, 797, 35 
Keller S. C., Mackey A. D., Da Costa G. S., 2011, ApJ, 731, 22

Keller S. C., Mackey A. D., Da Costa G. S., 2012, ApJ, 761, L5

King I., 1962, AJ, 67, 471

Kroupa P., 2002, Science, 295, 82

Mackey A. D., Gilmore G. F., 2003, MNRAS, 338, 85

Mackey A. D., Wilkinson M. I., Davies M. B., Gilmore G. F., 2008a, MNRAS, 386, 65

Mackey A. D., Broby Nielsen P., Ferguson A. M. N., Richardson J. C., 2008b, ApJ, 681, L17

Marigo P., Girardi L., Bressan A., Groenewegen M. A. T., Silva L., Granato G. L., 2008, A\&A, 482, 883

McLaughlin D. E., van der Marel R. P., 2005, ApJS, 161, 304

Milone A. P., Bedin L. R., Piotto G., Anderson J., 2009, A\&A, 497,755

Milone A. P., et al., 2015, MNRAS, 450, 3750

Milone A. P., Marino A. F., D'Antona F., Bedin L. R., Da Costa G. S., Jerjen H., Mackey A. D., 2016, MNRAS, 458, 4368

Mucciarelli A., Ferraro F. R., Origlia L., Fusi Pecci F., 2007, AJ, 133, 2053

Niederhofer F., Georgy C., Bastian N., Ekström S., 2015a, MNRAS, 453, 2070

Niederhofer F., Hilker M., Bastian N., Silva-Villa E., 2015b, A\&A, 575, A62

Niederhofer F., Bastian N., Kozhurina-Platais V., Hilker M., de Mink S. E., Cabrera-Ziri I., Li C., Ercolano B., 2016, A\&A, 586, A148

Piatti A. E., 2013, MNRAS, 430, 2358

Piatti A. E., 2014, MNRAS, 440, 3091

Piatti A. E., 2016, MNRAS,

Piatti A. E., Bastian N., 2016, preprint, (arXiv:1603.06891)

Piatti A. E., Bica E., 2012, MNRAS, 425, 3085

Piatti A. E., Geisler D., 2013, AJ, 145, 17

Piatti A. E., Keller S. C., Mackey A. D., Da Costa G. S., 2014, MNRAS, 444, 1425

Piatti A. E., et al., 2015, MNRAS, 454, 839

Pijloo J. T., Portegies Zwart S. F., Alexander P. E. R., Gieles M., Larsen S. S., Groot P. J., Devecchi B., 2015, MNRAS, 453, 605

Plummer H. C., 1911, MNRAS, 71, 460

Popescu B., Hanson M. M., Elmegreen B. G., 2012, ApJ, 751, 122

Rubele S., Girardi L., Kozhurina-Platais V., Goudfrooij P., Kerber L., 2011, MNRAS, 414, 2204

Rubele S., Girardi L., Kozhurina-Platais V., Kerber L., Goudfrooij P., Bressan A., Marigo P., 2013, MNRAS, 430, 2774

Salpeter E. E., 1955, ApJ, 121, 161

Spitzer Jr. L., Hart M. H., 1971, ApJ, 164, 399

Wilkinson M. I., Hurley J. R., Mackey A. D., Gilmore G. F., Tout C. A., 2003, MNRAS, 343, 1025

This paper has been typeset from a $\mathrm{TEX}_{\mathrm{E}} / \mathrm{LAT}_{\mathrm{E}} \mathrm{X}$ file prepared by the author. 